\section{Klassifikationssystem der American} Sleep Disorders Centers (ASDC)

\section{Helga Peter}

Marburg, Deutschland

\section{Definition}

Das Klassifikationssystem der Schlafstörungen der ASDC von 1979 machte die Hauptbeschwerden der Patienten zur Grundlage der Einteilung in vier diagnostische Hauptkategorien:

A. Ein- und Durchschlafstörungen (Disorders of Initiating and Maintaining Sleep, DIMS)
B. Störungen mit exzessiver Schläfrigkeit (Disorders od Excessive Somnolence, DOES)

C. Störungen des Schlaf-Wach-Rhythmus (Disorders of the Sleep-Wake Schedule)

D. Dysfunktionen in Verbindung mit Schlaf, Schlafstadien oder partiellem Erwachen, Parasomnien (Disorders of Sleep, Sleep Stages or Partial Arousals, Parasomnias)

Diese Einteilung wurde mit der Internationalen Klassifikation der Schlafstörungen der American Sleep Disorders Association (ASDA) von 1990 überholt.

Siehe „ „Diagnostische Klassifikationssysteme“. 